\title{
On the Periods of Certain Pseudorandom Sequences
}

\author{
By
}

\author{
Masahiko SATo*
}

\begin{abstract}
In [1], Rader et al. gave a fast method for generating pseudorandom sequences. Concerning these sequences, Moriyama et al. [2] made a research including the computational results by computers.

In this paper we shall study the periods of these sequences, and give an affirmative answer to the following conjecture presented in [2]:

"Let $k(n)$ be the maximum period of $n$-bit pseudorandom sequences generated by the Rader's method. Then $k(2 n)=2 k(n)$ for all $n$."

We shall also prove a number of algebraic properties of the periods, and give an efficient algorithm for computing $k(n)$.

We remark here that in this paper we are interested only in the algebraic properties of these sequences and not in the randomness of these sequences.
\end{abstract}

\section{§1. Introduction}

To make the present note self-contained, we begin with the definition of the pseudorandom sequences given by Rader et al.

An $n$-bit pseudorandom sequence $E=\left(E_{i}\right)_{i=0,1}, \ldots$ is defined inductively by:

$$
\left\{\begin{array}{l}
E_{0}=e_{0}, \\
E_{1}=e_{1}, \\
E_{i+2}=D\left(E_{i+1} \oplus E_{i}\right) \quad(i \geqq 0),
\end{array}\right.
$$

where $e_{0}$ and $e_{1}$ are given $n$-bit patterns, $\oplus$ denotes 'exclusive-or' of two $n$-bit patterns, and $D$ is the operator rotating the argument cyclically 1 bit to the right. For instance, if $n=3$ and $e_{0}=011, e_{1}=001$, we have: $E_{0}=011, E_{1}=001, E_{2}=001, E_{3}=000, E_{4}=100, \ldots, E_{14}=001, E_{15}=011$,

Communicated by S. Takasu, September 18, 1973.

* Graduate School, Department of Mathematics, Kyoto University, Kyoto. 
$E_{16}=001, \ldots$. We denote the $j$-th component of $E_{i}$ by $E_{i}(j-1)$. Thus $E_{i}=E_{i}(0) \ldots E_{i}(n-1)$. In the original paper by Rader et al., $D$ is replaced by $T_{p}$ which performs the $p$-bit cyclic rotation. Let us call this sequence $(n ; p)$-sequence. For the study of the period of the sequence, however, we have only to consider the case $p=1$. For, if $\operatorname{GCD}(p, n)=m \neq 1$, the sequence $\left(E_{i}\right)$ can be reduced to $m(n / m ; 1)$-sequences $\left(E_{i}^{j}\right)(j=1, \ldots, m)$, where $E_{i}^{j}(l)=E_{i}(j+(l-1) n / m)$. The period $k$ of the sequence $\left(E_{i}\right)$ is therefore obtained by $k=\operatorname{LCM}\left(k_{1}, \ldots, k_{m}\right)$, where $k_{j}$ is the period of $\left(E_{i}^{j}\right)$. If $\operatorname{GCD}(p, n)=1,\left(E_{i}\right)$ is isomorphic to the $(n ; 1)$ sequence $\left(E_{i}^{\prime}\right)$, where $E_{i}^{\prime}(j)=E_{i}\left(p^{j} \bmod n\right)$.

Now, let us consider the following sequence $\left(F_{i}\right)_{i=0,1}, \cdots$ of elements in $R$, where $R$ is a commutative ring with 1 and $f_{0}, f_{1}, x$ are fixed elements in $R$.

$$
\left\{\begin{array}{l}
F_{0}=f_{0}, \\
F_{1}=f_{1}, \\
F_{i+2}=x\left(F_{i+1}+F_{i}\right) \quad(i \geqq 0) .
\end{array}\right.
$$

Define the generating function $F \in R[[Y]]$ of $\left(F_{i}\right)$ as follows:

$$
F=\sum_{i=0}^{\infty} F_{i} Y^{i}
$$

From (2) and (3), by a simple computation, we obtain

$$
\begin{aligned}
F & =\left(f_{0}(1-x Y)+f_{1} Y\right) /\left(1-x Y-x Y^{2}\right) \\
& =\left(f_{0}+\left(f_{1}-f_{0} x\right) Y\right) \sum_{d=0}^{\infty} x^{d} Y^{d}(1+Y)^{d} .
\end{aligned}
$$

Hence,

$$
F_{i}=f_{0} \sum_{\substack{d+j=i \\
j \leqq d}}\left(\begin{array}{l}
d \\
j
\end{array}\right) x^{d}+\left(f_{1}-f_{0} x\right) \sum_{\substack{d+j+1=i \\
j \leqq d}}\left(\begin{array}{l}
d \\
j
\end{array}\right) x^{d}
$$

To see the relation between (1) and (2) more clearly, the following fact should be mentioned. The operator $D$ in (1) has the property that $D^{n}$ is the identity operation. So if we put 


$$
R=R_{n}=F_{2}[X] /\left(X^{n}-1\right)
$$

and $x=c(X)$, where $c: \boldsymbol{F}_{2}[X] \rightarrow \mathbb{F}_{2}[X] /\left(X^{n}-1\right)$ is the canonical mapping, then we can identify (2) and (1) under the following correspondence:

$$
\left.\begin{array}{l}
\text { an element of } R \\
\sum_{i=0}^{n-1} a_{i} X^{i} \quad\left(a_{i}=0,1\right)
\end{array}\right] \longleftrightarrow\left[\begin{array}{l}
\text { an } n \text {-bit pattern } \\
a_{0} a_{1} \ldots a_{n-1} \quad\left(a_{i}=0,1\right) \\
\text { multiplication by } X \longleftrightarrow \text { operation of } D
\end{array}\right.
$$

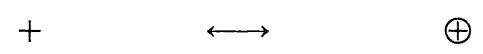

So in the following we shall consider (2) instead of (1).

To decompose $R_{n}$ into a direct sum, let

$$
X^{n}-1=\prod_{i=1}^{h} P_{i}^{e_{i}}
$$

be a factorization of $X^{n}-1$, where $P_{i}$ 's are distinct irreducible factors of $X^{n}-1$.

Since the derivative of $X^{n}-1$ is $n X^{n-1}, X^{s}-1=0$ has no repeated roots, i.e. $e_{i}=1$ for all $i$, when $n=s$ is odd. (In the following $s$ always denotes an arbitrary odd number.) Hence we have the following isomorphism.

$$
R_{s} \cong \boldsymbol{F}_{2}[X] /\left(P_{1}\right) \oplus \cdots \oplus \mathbb{F}_{2}[X] /\left(P_{h}\right)
$$

Now suppose $n$ is even and $n=2^{u} s$. Then since $X^{n}-1=X^{s 2 u}+1=$ $\left(X^{s}+1\right)^{2 u}$, we have

$$
X^{n}-1=P_{1}^{2 u} \ldots P_{h}^{2 u}
$$

Thus, we have

$$
R_{n} \cong \boldsymbol{F}_{2}[X] /\left(P_{1}^{2^{u}}\right) \oplus \cdots \oplus \boldsymbol{F}_{2}[X] /\left(P_{h}^{2^{u}}\right)
$$

\section{§2. Discussions in a Field}

Now let $P$ be any irreducible polynomial in $F_{2}[X]$ with degree d. Let us consider the relation (2) in the field $K=\mathbb{F}_{2}[X] /(P)=G F\left(2^{d}\right)$, 
taking $x \in K$ as the image of $X \in \boldsymbol{F}_{2}[X]$ by the natural mapping from $\mathbb{F}_{2}[X]$ to $K$.

Then we can naturally define a linear map $S: K^{2} \rightarrow K^{2}$ by:

$$
S=\left(\begin{array}{ll}
0 & 1 \\
x & x
\end{array}\right) .
$$

That is, $S$ is a function which maps $\left(\begin{array}{l}F_{i-1} \\ F_{i}\end{array}\right)$ to $\left(\begin{array}{l}F_{i} \\ F_{i+1}\end{array}\right)$. Hence,

$$
S^{i}\left(\begin{array}{c}
f_{0} \\
f_{1}
\end{array}\right)=\left(\begin{array}{c}
F_{i} \\
F_{i+1}
\end{array}\right) .
$$

Since $\operatorname{det} S=x \neq 0, \quad S$ is in $G L(2, K)$. So the group $G=<S>\subset$ $G L(2, K)$ acts on $K^{2}$ from left in a natural way. For any $f \in K^{2}$, we put $k_{K}(\boldsymbol{f})=k(\boldsymbol{f})=|G \boldsymbol{f}|$, namely the cardinality of the $G$-orbit containing $f$. Clearly, $k(f)$ is the period of the sequence (2) for the initial value $\boldsymbol{f}=\left(\begin{array}{l}f_{0} \\ f_{1}\end{array}\right)$.

As is well-known, $|G \boldsymbol{f}|=|G| /\left|G_{\boldsymbol{f}}\right|$, where $G_{\boldsymbol{f}}$ is the stabilizer of $\boldsymbol{f}$. We have therefore

$$
k(f)|| G \mid \quad\left(\text { for all } f \in K^{2}\right) .
$$

If we put $k=k(\boldsymbol{f})$, we have

$$
\begin{aligned}
& S^{k}(\boldsymbol{f})=\boldsymbol{f} \text { and } \\
& S^{k}(S \boldsymbol{f})=S \boldsymbol{f} .
\end{aligned}
$$

So, if $\{\boldsymbol{f}, S \boldsymbol{f}\}$ is a basis of $K^{2}$, we have $S^{k}=\left(\begin{array}{ll}1 & 0 \\ 0 & 1\end{array}\right)$. This, combined with (11), means $k(\boldsymbol{f})=|G|$. $1 \neq 0$.

Thus, the initial value $\left(\begin{array}{l}0 \\ 1\end{array}\right)$ gives the maximum period, since $\left|\begin{array}{ll}0 & 1 \\ 1 & x\end{array}\right|=$

Remark. The above argument remains valid even if we take as $P$ any non-constant polynomial in $\boldsymbol{F}_{2}[X]$ whose constant term is not 0 , merely by replacing ' $\neq 0$ ' by 'is invertible' in two places above.

Now, $\boldsymbol{f}$ and $\boldsymbol{S} \boldsymbol{f}$ are linearly dependent iff $\boldsymbol{f}$ is an eigenvector of $S$. Since the eigenpolynomial of $S$ is 


$$
E(t)=t^{2}+x t+x
$$

we have the following

Theorem 1. If $E(t)=0$ has no roots in $K$, then every orbit other than $\left\{\left(\begin{array}{l}0 \\ 0\end{array}\right)\right\}$ has the same period $k=|G|$.

Corollary 2. $|G| \mid 2^{2 d}-1$.

Let $\alpha, \beta$ be the roots of $E(t)=0$ in the algebraic closure $\bar{K}$ of $K$. Let $K^{\prime}=K(\alpha, \beta)$. Since $\alpha+\beta=x \neq 0, \alpha$ and $\beta$ are distinct. Since $\alpha \beta=$ $x \neq 0, \alpha$ and $\beta$ are not 0 . Thus for some $U \in G L\left(2, K^{\prime}\right)$, we have

$$
S=U\left(\begin{array}{ll}
\alpha & 0 \\
0 & \beta
\end{array}\right) U^{-1}
$$

If $K^{\prime} \neq K$ then $K^{\prime}$ is an extension field of degree 2 over $K$. Hence $K^{\prime} \cong G F\left(2^{2 d}\right)$. Since $\alpha$ and $\beta$ are conjugate over $K$, we see $|\alpha|=|\beta|$, where $|\alpha|,|\beta|$ are the orders of $\alpha, \beta$ as elements of the multiplicative group of $K^{\prime}$. And, since $\alpha$ is not in $K,|\alpha|$ can not divide $\left|K^{*}\right|$, where $K^{*}$ is the multiplicative group of $K$. From (13) and the above arguments, the following theorem can be obtained.

Theorem 3. (i) If $E(t)=0$ is unsolvable in $K$, then

$$
\begin{aligned}
& |G|=|\alpha|=|\beta| \mid 2^{2 d}-1, \quad \text { and } \\
& |G| \Varangle 2^{d}-1 .
\end{aligned}
$$

(ii) If $E(t)=0$ is solvable in $K$, then

$$
|G|=\operatorname{LCM}(|\alpha|,|\beta|) \mid 2^{d}-1 \text {, and }
$$

the period of $\boldsymbol{f} \neq 0$ is

$$
k(\boldsymbol{f})= \begin{cases}|\alpha| & (\text { if } \boldsymbol{S} \boldsymbol{f}=\alpha \boldsymbol{f}) \\ |\beta| & (\text { if } \boldsymbol{S} \boldsymbol{f}=\beta \boldsymbol{f}) \\ |G| & \text { (otherwise) }\end{cases}
$$

Now, let us compute the general term of the sequence $\left(F_{i}\right)$. As 
the transformation matrix $U$ in (13), we may take

$$
\begin{aligned}
& U=\left(\begin{array}{ll}
1 & 1 \\
\alpha & \beta
\end{array}\right), \\
& U^{-1}=\frac{1}{\alpha+\beta}\left(\begin{array}{ll}
\beta & 1 \\
\alpha & 1
\end{array}\right) .
\end{aligned}
$$

Hence,

$$
\begin{aligned}
S^{i} & =U\left(\begin{array}{ll}
\alpha^{i} & 0 \\
0 & \beta^{i}
\end{array}\right) U^{-1} \\
& =\frac{1}{\alpha+\beta}\left(\begin{array}{ll}
\alpha^{i} \beta+\alpha \beta^{i} & \alpha^{i}+\beta^{i} \\
\alpha^{i+1} \beta+\alpha \beta^{i+1} & \alpha^{i+1}+\beta^{i+1}
\end{array}\right) .
\end{aligned}
$$

Hence, by (10) and (15),

$$
F_{i}=\frac{1}{\alpha+\beta}\left(a \beta\left(\alpha^{i-1}+\beta^{i-1}\right) f_{0}+\left(\alpha^{i}+\beta^{i}\right) f_{1}\right)
$$

\section{§3. Proof of the Conjecture}

Let us now return to the original problem and consider the case $n=s$. The relation (7) may be written as

$$
R_{s} \cong K_{1} \oplus \cdots \oplus K_{h}
$$

Consider the sequence (2) in the ring $R_{s}$, and fix an initial value $\left(\begin{array}{l}f_{0} \\ f_{1}\end{array}\right) \in$ $R_{s}^{2}$. The above isomorphism is induced from the natural ring homomorphisms $\varphi_{i}: R_{s} \rightarrow K_{i}$. Hence the following relation clearly holds.

$$
k_{R_{s}}\left(\begin{array}{c}
f_{0} \\
f_{1}
\end{array}\right)=\operatorname{LCM}\left(k_{K_{1}}\left(\begin{array}{c}
\varphi_{1}\left(f_{0}\right) \\
\varphi_{1}\left(f_{1}\right)
\end{array}\right), \ldots, k_{K_{h}}\left(\begin{array}{c}
\varphi_{h}\left(f_{0}\right) \\
\varphi_{h}\left(f_{1}\right)
\end{array}\right)\right)
$$

Now, take any non-constant polynomial $P$ in $\boldsymbol{F}_{2}[X]$ whose constant term is not 0 , and consider the sequences (2) in two rings

$$
Q_{1}=\boldsymbol{F}_{2}[X] /(P) \text { and } Q_{2}=\boldsymbol{F}_{2}[X] /\left(P^{2}\right)
$$

We examine the relation between the periods of two sequences in $Q_{1}$ 
and $Q_{2}$ for the initial values $\left(\begin{array}{l}0 \\ 1\end{array}\right) \in Q_{1}^{2}$ and $\left(\begin{array}{l}0 \\ 1\end{array}\right) \in Q_{2}^{2}$, respectively. To this end, we consider the sequence (2) in $\boldsymbol{F}_{2}[X]$ putting $f_{0}=0$ and $f_{1}=1$. Let $k=k_{Q_{1}}\left(\begin{array}{l}0 \\ 1\end{array}\right)$. Then for some $A_{1}, A_{2}, A_{3}, A_{4} \in \boldsymbol{F}_{2}[X]$, we have

$$
S^{k}=\left(\begin{array}{ll}
A_{1} P+1 & A_{2} P \\
A_{3} P & A_{4} P+1
\end{array}\right)
$$

Hence,

$$
S^{2 k} \equiv\left(\begin{array}{cc}
1 & 0 \\
0 & 1
\end{array}\right) \quad\left(\bmod P^{2}\right)
$$

Hence, by (11)

$$
k_{Q_{2}}\left(\begin{array}{c}
0 \\
1
\end{array}\right) \mid 2 k
$$

On the other hand, if $l=k_{Q_{2}}\left(\begin{array}{l}0 \\ 1\end{array}\right)<k$ then, since $S^{l} \equiv\left(\begin{array}{ll}1 & 0 \\ 0 & 1\end{array}\right)\left(\bmod P^{2}\right)$, we have $S^{l} \equiv\left(\begin{array}{ll}1 & 0 \\ 0 & 1\end{array}\right)(\bmod P)$. This is a contradiction. Thus,

$$
k_{Q_{2}}\left(\begin{array}{l}
0 \\
1
\end{array}\right)=k_{Q_{1}}\left(\begin{array}{l}
0 \\
1
\end{array}\right) \quad \text { or } k_{Q_{2}}\left(\begin{array}{l}
0 \\
1
\end{array}\right)=2 k_{Q_{1}}\left(\begin{array}{l}
0 \\
1
\end{array}\right) \text {. }
$$

Now, let $k(n)$ be the maximum period of the $n$-bit random sequence (1). Then since the initial pattern $\left(\begin{array}{l}0 \\ 1\end{array}\right)$ gives the maximum period, we have

$$
k(n)=k_{R n}\left(\begin{array}{l}
0 \\
1
\end{array}\right)
$$

By (19), (20), and the fact that $\left(X^{n}+1\right)^{2}=X^{2 n}+1$, we have

$$
k(2 n)=k(n) \quad \text { or } \quad k(2 n)=2 k(n) .
$$

We now prove that the case $k(2 n)=k(n)$ never occurs.

Theorem 4. $k(2 n)=2 k(n)$. 
Proof. If $n=s$ then by (17),

$$
k(s)=\operatorname{LCM}\left(k_{K_{1}}\left(\begin{array}{l}
0 \\
1
\end{array}\right), \ldots, k_{K_{h}}\left(\begin{array}{l}
0 \\
1
\end{array}\right)\right) .
$$

Then by Theorem 3 we see that $k_{i}=k_{K_{i}}\left(\begin{array}{l}0 \\ 1\end{array}\right)$ is odd for all $1 \leqq i \leqq h$. Hence $k(s)$ is odd. If $n=2^{m} s(m \geqq 0)$, then by (19) and (22),

$$
\begin{aligned}
k(n) & =\operatorname{LCM}\left(2^{m_{1}} k_{1}, \ldots, 2^{m_{h}} k_{h}\right) \quad\left(0 \leqq m_{i} \leqq m\right) \\
& =2^{\max \left\{m_{1}, \ldots, m_{h}\right\}} k(s) .
\end{aligned}
$$

Hence, if we can prove that

$$
m=\max \left\{m_{1}, \ldots, m_{h}\right\}
$$

then we have

$$
k\left(2^{m} s\right)=2^{m} k(s) \quad(m \geqq 0) .
$$

This yields immediately Theorem 4 .

Now, since $X+1$ is an irreducible factor of $X^{s}+1$, we may assume $K_{1}=F_{2}[X] /(X+1)$. So, to prove (24), we have only to show that $m_{1}=m$. Comparing (24), with (23), we see that $m_{1}=m$ iff $k_{R_{2} m}\left(\begin{array}{l}0 \\ 1\end{array}\right)=$ $2^{m} k_{R_{1}}\left(\begin{array}{l}0 \\ 1\end{array}\right)$. Hence we have only to prove

$$
\begin{aligned}
k\left(2^{m}\right) & =2^{m} k(1) \\
& =2^{m} 3 .
\end{aligned}
$$

Thus (25) is reduced to its special case (26).

Now, to show (26), let us consider the sequence (2) in the field $\overline{F_{2}(X)}$, where $\overline{F_{2}(X)}$ is the algebraic closure of the field $\boldsymbol{F}_{2}(X)$ which is the quotient field of $\boldsymbol{F}_{2}[X]$. If we set $f_{0}=0$ and $f_{1}=1$, then by (16),

$$
F_{i}=\left(\alpha^{i}+\beta^{i}\right) /(\alpha+\beta),
$$

where $\alpha$ and $\beta$ are the two roots of $E(t)=t^{2}+X t+X=0$ in $\overline{F_{2}(X)}$. Since $\alpha+\beta=X$, we have 


$$
F_{2^{m}}=\left(\alpha^{2^{m}}+\beta^{2^{m}}\right) /(\alpha+\beta)=(\alpha+\beta)^{2^{m}} /(\alpha+\beta)=X^{2^{m-1}} .
$$

Now, (26) trivially holds for $m=0$. For $m \geqq 1$, we prove $k\left(2^{m}\right)=$ $2^{m} 3$ assuming $k\left(2^{m-1}\right)=2^{m-1} 3$. Let us suppose that $k\left(2^{m}\right) \neq 2^{m} 3$. Then, by (21),

$$
k\left(2^{m}\right)=k\left(2^{m-1}\right)=2^{m-1} 3
$$

Hence by (28),

$$
\begin{aligned}
X^{2^{m+1}-1} & =F_{2^{m+1}} \\
& =F_{2^{m-1}+2^{m-1} 3} \\
& \equiv F_{2^{m-1}} \quad\left(\bmod X^{2^{m}}+1\right) \\
& \equiv X^{2^{m-1}-1} \quad\left(\bmod X^{2^{m}}+1\right) .
\end{aligned}
$$

On the other hand,

$$
\begin{aligned}
X^{2^{m+1}-1} & =X^{2^{m}} X^{2^{m-1}} \\
& \equiv X^{2^{m-1}} \quad\left(\bmod X^{2^{m}}+1\right) .
\end{aligned}
$$

This is a contradiction. Theorem 4 is now proved.

\section{§4. Other Properties of $k(n)$}

Besides that $k(2 n)=2 k(n), k(n)$ has many properties. In this $\S$ we prove some of them. Theorem 4 established in the last $\S$ plays an important rôle. Using these properties we give an algorithm for calculating $k(n)$ which is more efficient than the straightforward algorithm.

Theorem 5. If $m \mid n$ then $k(m) \mid k(n)$.

Proof. First suppose $m$ and $n$ are both odd. Then if $P$ is an irreducible polynomial dividing $X^{m}+1, P$ divides $X^{n}+1$. Hence by (22), we see $k(m) \mid k(n)$. Now consider the general case. Suppose $m=2^{u_{1}} s_{1}$ and $n=2^{u_{2}} s_{2}$, where $s_{1}, s_{2}$ are odd. Then, $k(m)=2^{u_{1}} k\left(s_{1}\right)$ and $k(n)=$ $2^{u_{2}} k\left(s_{2}\right)$ by Theorem 4. If $m \mid n$, then $u_{1} \leqq u_{2}$ and $s_{1} \mid s_{2}$. Hence $k(m) \mid$ 
$k(n)$, since $k\left(s_{1}\right) \mid k\left(s_{2}\right)$.

Corollary 6. $3 \mid k(n)$.

Proof. $1 \mid n$ and $k(1)=3$

Theorem 7. $n \mid k(n)$.

Proof. First suppose $n=s$. Let $\zeta$ be a primitive $s$-th root of 1 . Then $L_{s}=\boldsymbol{F}_{2}(\zeta)$ is the splitting field of $X^{s}+1=0$. Let $d(s)=\left[L_{s}: \boldsymbol{F}_{2}\right]$. Let $P \in \mathbb{F}_{2}[X]$ be the minimal polynomial of $\zeta$. Then $P=(X-\zeta)(X-$ $\left.\zeta^{2}\right) \cdots\left(X-\zeta^{2 d(s)-1}\right)$. Hence $d(s)$ is the least positive integer such that $s \mid 2^{d(s)}-1$. Since $\zeta, \zeta^{2}, \ldots, \zeta^{d(s)-1}$ are the roots of $X^{s}+1=0, P \mid X^{s}+1$. Thus $P$ is an irreducible factor of $X^{s}+1$. Consider the sequence (2) in the field $L_{s}$, where we set $x=\zeta$. From (22), we have

$$
k_{L_{s}}\left(\begin{array}{l}
0 \\
1
\end{array}\right) \mid k(s)
$$

Let $k=k_{L_{s}}\left(\begin{array}{l}0 \\ 1\end{array}\right)$. From (13) we see that

$$
\alpha^{k}=\beta^{k}=1,
$$

where $\alpha, \beta$ are the roots of $t^{2}+\zeta t+\zeta=0$. Hence $\zeta^{k}=(\alpha \beta)^{k}=1$. Hence

$$
s \mid k
$$

By (29) and (30), we have $s \mid k(s)$. The case when $n$ is even can be proved by using Theorem 4 .

Theorem 8. $k(s) \mid 2^{2 d(s)}-1$

Proof. Since $L_{s}$ is the splitting field of $X^{s}+1=0$, we may consider that each $K_{i}=F_{2}[X] /\left(P_{i}\right)$ is a subfield of $L_{s}$. Hence by (11) and Theorem 3, we have $k_{K_{i}}\left(\begin{array}{l}0 \\ 1\end{array}\right) \mid 2^{2 d(s)}-1$. Hence, by (22), we have $k(s) \mid 2^{2 d(s)}$ -1 .

Theorem 3 and the above proof show that if $E_{i}(t)=t^{2}+\zeta^{i} t+\zeta^{i}=0$ 
is solvable in $L_{s}$ for all $1 \leqq i \leqq s$, then $k(s) \mid 2^{d(s)}-1$. But the following theorem tells that this case does not occur. Before proving the theorem, we give an example.

Let $s=7$. Then $d(s)=3$. The factorization of $X^{7}+1$ is $(X+1)\left(X^{3}+\right.$ $X+1)\left(X^{3}+X^{2}+1\right)$. Let $\zeta$ be a root of $X^{3}+X^{2}+1=0$. Then since $\left(\zeta^{2}\right)^{2}+\zeta\left(\zeta^{2}\right)+\zeta=\zeta\left(\zeta^{3}+\zeta^{2}+1\right)=0, E_{i}(t)=0$ is solvable in $L_{s}$ for $i=1,2,4$. But, for other $i$ 's, $E_{i}(t)=0$ is unsolvable in $L_{s}$.

Theorem 9. If $d<2 d(s)$ then $k(s) \times 2^{d}-1$

Proof. Consider the sequence (2) in the field $\overline{F_{2}(X)}$, setting $f_{0}=0$, $f_{1}=1$. Then by (27), since $\alpha+\beta=X$,

$$
F_{2 i}=\left(\alpha^{2 i}+\beta^{2 i}\right) /(\alpha+\beta)=X F_{i}^{2} .
$$

Let

$$
G_{m}=F_{2^{m}-1}
$$

Then by (31), $F_{2^{m+1-2}}=X G_{m}^{2}$. By (28), $F_{2^{m+1}}=X^{2^{m+1}-1}$. Since $X^{2^{m+1}-1}$ $=F_{2^{m+1}}=X\left(F_{2^{m+1-1}}+F_{2^{m+1-2}}\right)=X\left(G_{m+1}+X G_{m}^{2}\right)$, we have

$$
G_{m+1}=X^{2^{m+1}-2}+X G_{m}^{2} .
$$

Using (33) we can prove by induction that

$$
G_{m}=\sum_{j=0}^{m-1} X^{2^{m-2 j-1}}
$$

If $k(s) \mid 2^{d}-1$ then we have $G_{d} \equiv 0\left(\bmod X^{s}+1\right)$. Hence, if we write $G_{d}$ in the form of (34), there must be some $0<j<d$ such that

$$
X^{2^{d-2 J-1}} \equiv X^{2^{d+20-1}} \quad\left(\bmod X^{s}+1\right) .
$$

Hence

$$
2^{d}-2^{j}-1 \equiv 2^{d}-2 \quad(\bmod s)
$$

Or

$$
2^{j} \equiv 1 \quad(\bmod s)
$$


Since $d(s)$ is the least positive integer such that $s \mid 2^{d(s)}-1$, we have $j \geqq$

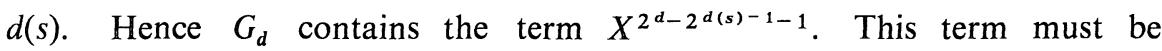
canceled by some term of the form $X^{2^{d-2 J-1}}$, where $d(s)-1<j<d$. Hence

$$
2^{d}-2^{d(s)-1}-1 \equiv 2^{d}-2^{j}-1 \quad(\bmod s) .
$$

Or

$$
2^{d(s)-1} \equiv 2^{j} \quad(\bmod s)
$$

Or

$$
1 \equiv 2^{j+1} \quad(\bmod s)
$$

Since $j+1>d(s)$, we must have $j+1 \geqq 2 d(s)$. This contradicts with the fact that $2 d(s)>d>j$.

Putting Theorems 8 and 9 together, we have the following

Corollary 10. $d(k(s))=2 d(s)$.

Let us now consider the sequence (2) in $\overline{F_{2}(X)}$, setting $f_{0}=0, f_{1}=1$. By (2) and (27), we have

$$
\left\{\begin{array}{l}
F_{2 i}=X F_{i}^{2} \\
F_{2 i+1}=F_{i+1}^{2}+X F_{i}^{2} .
\end{array}\right.
$$

Clearly these equations also hold in $R_{n}$ (for the initial values $f_{0}=0$, $f_{1}=1$ ). Then, for any given $m$, by the iterative use of (35), we can easily calculate the value of $F_{m}$ (in $R_{n}$ ). Now, since the candidates $m$ for the period $k(n)$ can be confined to a reasonable number by using Theorems 5-9, we can compute $k(n)$ pretty easily. Indeed, sometimes we can determine the period without any computations:

Theorem 11. If $f$ is a Fermat prime then $k(f-2)=(f-2) f$.

Proof. Let $f=2^{e}+1$. Then $d(f-2)=d\left(2^{e}-1\right)=e$. By Theorem 7, $f-2 \mid k(f-2)$. By Theorem 8, $k(f-2) \mid(f-2) f$. By Theorem 9, $k(f-2) \chi$ $f-2$. Therefore, since $f$ is a prime, $k(f-2)=(f-2) f$. 


\section{References}

[1] Rader, C. M., Rabiner, L. R. and Schafer, R. W., A fast method of generating digital random numbers, Bell System Tech. J., 148 (1970), 2203-2310.

[2] Moriyama, S. and Kitamura, S., On a fast method of generating random numbers (in Japanese), Joho-shori, 14 (1973), 15-22. 
